\title{
Effect of combinational arginase and canavanine treatment on normal human peripheral blood lymphocytes in vitro
}

\author{
OLEH CHEN $^{1,2}$, BOHDANNA KAVALETs ${ }^{1,2}$, MARINA BARSKA ${ }^{1}$, LILIANNa LYNIV ${ }^{1}$, \\ OLENA VOVK ${ }^{1}$, NATALIA SYBIRNA ${ }^{2}$, OLEH STASYK ${ }^{1}$ \\ ${ }^{1}$ Department of Cell Signaling, Institute of Cell Biology of NAS of Ukraine, Lviv, Ukraine \\ ${ }^{2}$ Department of Biochemistry, Ivan Franko Lviv National University, Lviv, Ukraine
}

\begin{abstract}
Recombinant human arginase (rhARG)-based therapy to restrict arginine availability for malignant cells is a promising strategy to treat some cancers, including those of hematological origin. In the present study, we evaluated the action of combined rhARG treatment with canavanine (Cav) on proliferative potential and functional state of human peripheral blood lymphocytes (PBL) in vitro. We demonstrated that Cav in $0.1 \mathrm{mM}$ concentration alone had no significant effect on proliferation and functional activity of normal activated PBL. Combined rhARG and Cav treatment inhibited proliferation and slightly promoted apoptosis in activated PBL but did not affect resting cells. However, the action of both drugs affected the ability of $\mathrm{T}$ cells for antigen activation. These results are important for further translational research on arginine-deprivation therapy.
\end{abstract}

Keywords: arginase, canavanine, arginine deprivation, peripheral blood lymphocytes

\section{INTRODUCTION}

Cytotoxicity of anticancer drugs toward normal cells is one of the most serious problems of chemotherapy, leading to side effects such as immunosupression, anemia, blood clotting and others [3]. Therefore, the immunotoxicological evaluation of novel therapeutic agents is a critical component in the development of new therapies.

L-arginine plays a central role in regulating several biological systems, including the immune response, and is essential for normal $\mathrm{T}$ lymphocytes proliferation and function [1]. Some authors showed that activated primary $T$ cells cultured in the medium deprived of arginine were arrested in the G0-G1 phase of the cell cycle, exhibited decreased proliferation, low expression of T-cell receptor CD3 $\zeta$ chain and impaired production of cytokines, but did not induce apoptosis $[11,12]$. On the other hand, abrogated proliferation and induction of apoptosis was shown for Jurkat leukemic cells cultured in the absence of L-arginine. [2].

Pharmacological deprivation of arginine with the recombinant arginine-degrading enzymes, arginine deiminase

\footnotetext{
Corresponding author

* Department of Cell Signaling, Institute of Cell Biology of NASU,

Drahomanov Str., 14/16, 79005, Lviv, Ukraine

e-mail: stasyk@cellbiol.lviv.ua
}

or rhARG, is a new strategy to treat some cancers, which is currently under clinical trials $[17,20]$. Another drugCav, a toxic arginine analogue of plant origin, is a promising candidate for augmenting the antineoplastic effects of arginine starvation [18].

L-Cav, a non-protein amino acid, is produced by a variety of leguminous plants and replaces arginine when incorporated into newly synthesized proteins in proliferatively active cells, resulting in the formation of aberrant, non-functional proteins $[13,19]$. The cytotoxic effect of Cav has been shown in tumor cells both in vitro and in vivo [15, 16], as a single agent [9] or in combination with chemotherapeutic drugs or radiotherapy [7, 14].

In this study, we addressed the hypothesis that, under arginine deprivation, Cav cytotoxic concentrations should be profoundly lower for cancer cells but not normal immune cells, and analyzed the effect of combined rhARG treatment with Cav on viability and functional activity of normal human PBL.

\section{MATERIALS AND METHODS}

Chemicals. RPMI-1640 cell culture medium, fetal bovine serum (FBS), Histopaque 1077, gentamycin, glutamine, phytohemagglutinin (PHA), L-canavanine sulphate, trypan blue, 3-(4,5-Dimethylthiazol-2-yl)-2,5diphenylte-

DOI: $10.12923 /$ j.2084-980X/26.4/a.07 
trazolium bromide (MTT) and fluorescent dyes - 4',6diamidino-2-phenyindole dilactate (DAPI), Annexin V-FITC, propidium iodide were obtained from SigmaAldrich (USA). Recombinant interleukin-2 (rIL-2) was purchased from Millipore Corporation (USA). rhARG with concentration $2 \mathrm{U} / \mathrm{mL}$ used for arginine deprivation was constructed and purified in ICB of NAS of Ukraine. Griess reagent and other remaining chemicals were produced by Synbias (Ukraine).

Separation and cultivation of blood cells. PBL were isolated from heparinized whole blood of healthy donors and separated by density Histopaque 1077 according to instruction. Mononuclear cells were maintained in RPMI-1640 medium supplemented with 10\% FBS, 300 $\mathrm{mg} / \mathrm{L}$ glutamine and $50 \mu \mathrm{g} / \mathrm{mL}$ gentamycin in a humidified $5 \% \mathrm{CO}_{2}$ incubator at $37^{\circ} \mathrm{C}$. Proliferation of $\mathrm{T}$ lymphocytes was induced by incubation of PBL for 4 days with $0.5 \mu \mathrm{g} / \mathrm{mL}$ PHA and $20 \mathrm{U} / \mathrm{ml}$ rIL-2 in culture medium. Resting PBL were cultured in the presence of 20 $\mathrm{U} / \mathrm{mL}$ rIL-2 only. Resting and proliferatively active PBL were treated with $2 \mathrm{U} / \mathrm{mL}$ purified rhARG and/or Cav. The number of viable cells was counted with trypan blue dye solution in hemocytometer. Concentration- and time-dependent Cav cytotoxicity was determined using the standard MTT assay.

Measurement of $\mathrm{NO}$ production. The amount of NO released by activated PBL was estimated by measuring the accumulation of the terminal metabolite of $\mathrm{NO}$, nitrite $\left(\mathrm{NO}_{2}^{-}\right)$, in the culture medium by using the Griess method as previously described [8].

Light and fluorescent microscopy. Cell morphology and chromatin structure of resting and activated PBL were visualized in differential interference contrast (DIC) microscopy with DAPI $(1 \mu \mathrm{g} / \mathrm{mL})$ fluorescence dye staining and light microscopy with Romanowsky-Giemsa staining. Apoptosis detection was performed using the Annexin V/PI fluorochromes by fluorescent microscopy as described above [5].

Statistical analysis. All experiments were repeated at least three times. Levels of significant differences between groups were determined by the Student's t-test. P values less then 0.05 were considered statistically significant.

\section{RESULTS AND DISCUSSION}

In this study, two experimental models of normal human lymphocytes, the mitogen PHA-activated and primary isolated resting cells, were used. We examined proliferative potential and viability of immune cells under arginine deprivation alone or in combination with Cav. For this purpose, cells were incubated in cell culture medium supplemented with rhARG in concentration of 2.0 $\mathrm{U} / \mathrm{mL}$ and a number of viable cells was counted by the trypan blue dye exclusion assay. As shown in Fig.1 A,
rhARG significantly inhibited proliferation of PHA-activated PBL but did not drastically decrease cell viability $(\mathrm{p}<0.05)$. Also, arginine restriction by rhARG had no effect on normal resting PBL viability (Fig. 1 B). We further utilized plant arginine analogue - $\mathrm{Cav}$ at concentration of $0.1 \mathrm{mmol} / \mathrm{L}$, alone or in combination with rhARG, and evaluated its cytotoxic activity to PBL. We previously reported that this concentration of Cav was cytotoxic for several types of cancer cells under argininedeprived conditions, but not in an arginine-sufficient medium [18]. In this work, we showed that this concentration of Cav in arginine-rich medium had no significant effect on proliferatively active normal cells and slightly suppressed PHA-stimulated proliferation of PBL $(p<0.05)$. As shown in Fig. 1 A, incubation of PHA-activated PBL with Cav in the presence of rhARG caused a decrease in cell viability. However, there was no statistically significant difference observed in the amount of viable cells in resting PBL (Fig. $1 \mathrm{~B}$ ) under combined treatment $(p<0.05)$. Therefore, normal resting PBL were more resistant to combined drugs action.

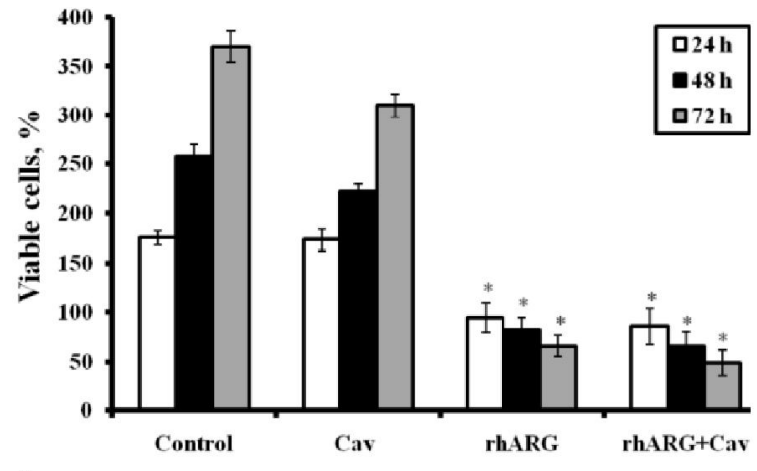

A

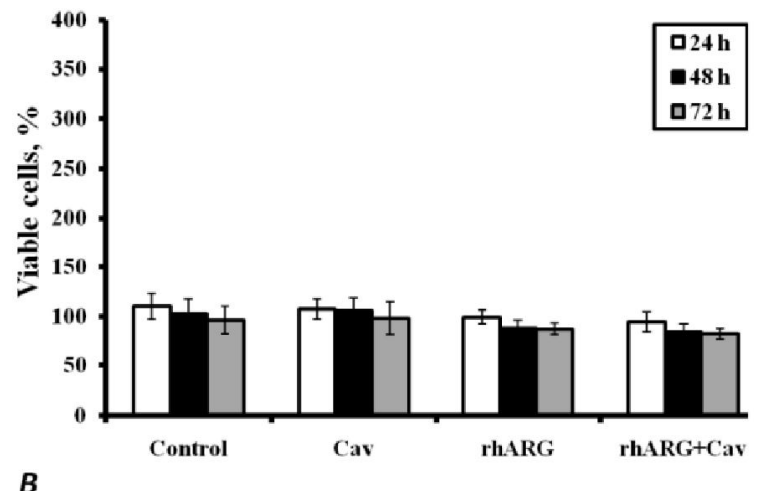

Fig. 1. Effect of combined rhARG and Cav treatment on viability of PHA-activated (A) and resting PBL (B) cultivated in control complete medium (Control), medium with $0.1 \mathrm{mM}$ canavanine (Cav), medium with rhARG, $2 \mathrm{U} / \mathrm{mL}$ (rhARG), and medium with rhARG and $0.1 \mathrm{mM}$ canavanine (rhARG+Cav). Cell numbers were determined by the trypan blue exclusion test for $24,48,72 \mathrm{~h}$. ${ }^{*} \mathrm{p} \leq 0.05$ as compared with the untreated cells.

We next examined in details the Cav inhibitory concentration (the concentration that causes death of $50 \%$ of 
cells, IC50) for resting and proliferatively active cells upon different culture conditions. There were no differences observed $(\mathrm{p}<0.05)$ between the viability of the cells in the presence of $\mathrm{Cav}$ at concentrations up to $0.1 \mathrm{mM}$ for PHA-activated PBL and up to $1 \mathrm{mM}$ for resting cells during 72 hours of the treatment. Cav was cytotoxic for proliferatively active normal lymphocytes at the concentration range from 1 to $10 \mathrm{mM}$, and above $10 \mathrm{mM}$ for resting cells. The appropriate Cav IC50 values for PBL are provided in Table 1. We also observed that Cav IC50 data for PHA-activated PBL did not differ drastically between complete medium and medium with rhARG. The IC50 values were $10,7.0 \pm 1.2$ and $4.5 \pm 0.74 \mathrm{mM}$ at 24,48 , and $72 \mathrm{~h}$ in arginine-rich medium and 10, 6.3 \pm 1.1 and $1.1 \pm 0.3 \mathrm{mM}$ in the medium supplemented with rhARG, respectively. As shown in Table 1, Cav-mediated cytotoxicity was significantly lower for the resting PBL. Accordingly, IC50 values were significantly higher: more than $20 \mathrm{mM}$ in arginine-containing medium and $17 \pm 1.4$ $\mathrm{mM}$ under arginine deprivation at $72 \mathrm{~h}$. Apparently, Cav in low concentrations (below $100 \mu \mathrm{M}$ ) is non-toxic for the tested immune cells.
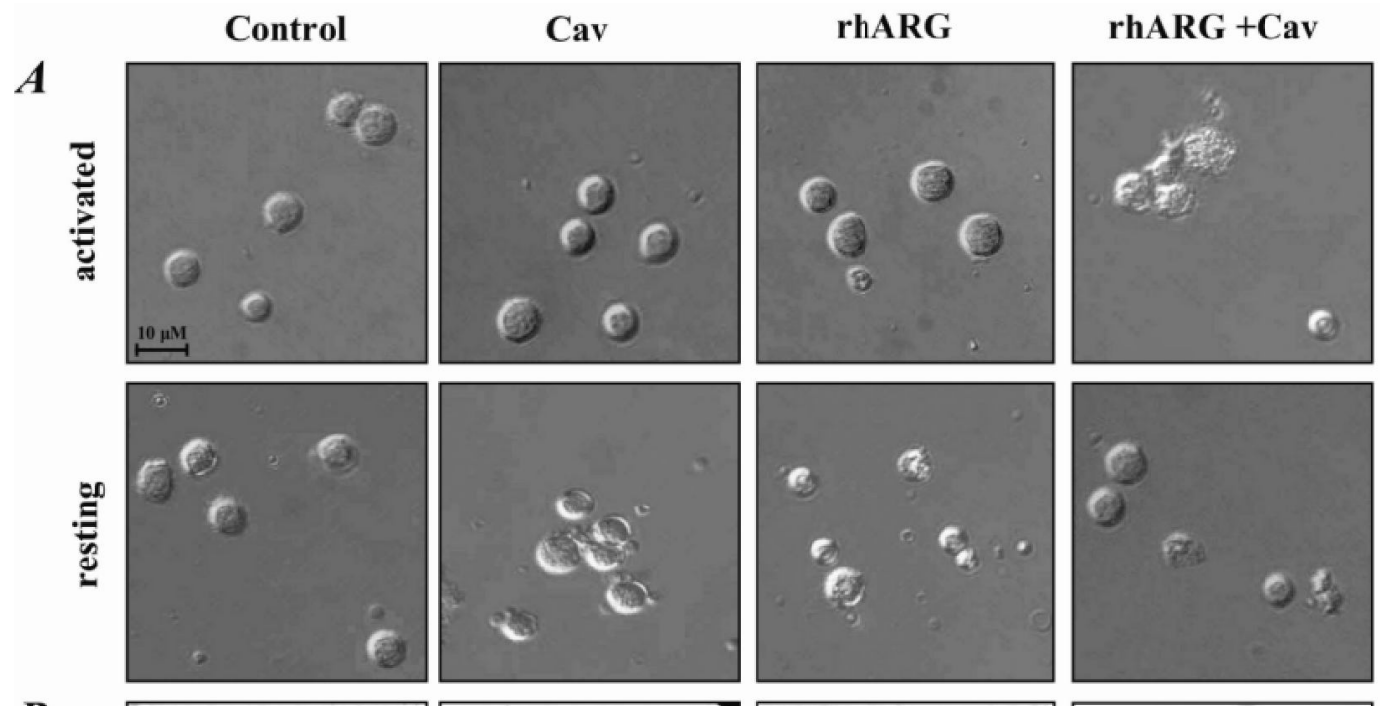

B
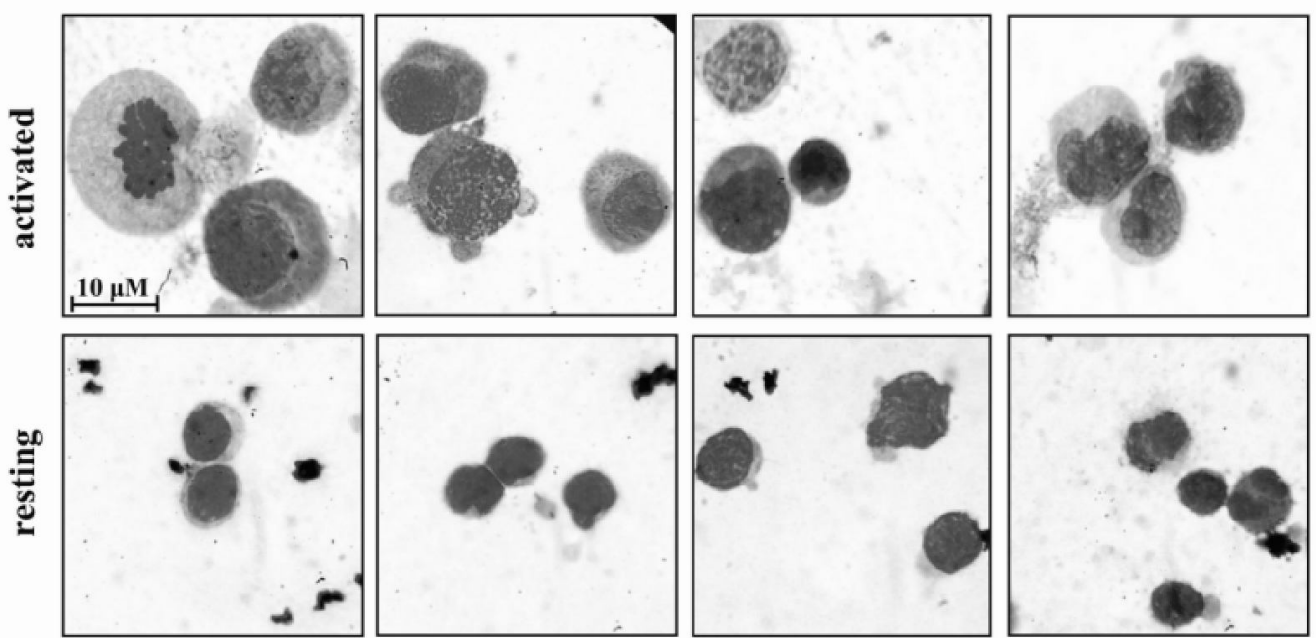

Fig. 2. Effect of the combined rhARG and Cav treatment ( $48 \mathrm{~h}$ ) on morphology of PHA-activated and resting PBL stained with DAPI fluorescence dye (A, x 600) or Romanovsky-Giemsa (B, x 1500). Merged images of DAPI fluorescence were obtained using a DIC microscopy with fluorescence. 
Next, we analyzed the phosphatidylserine externalization with Annexin V-FITC and propidium iodide double staining of experimental models of PBL (Fig. 3). We observed no significant changes in the number of apoptotic cells upon combined $72 \mathrm{~h}$-long treatment with rhARG and Cav relative to rhARG treatment alone in resting cells ( $p$ 0.05). As shown in Fig. 3, minimal signs of apoptosis in PHA-activated cells were observed after $72 \mathrm{~h}$ of culture with rhARG alone (17\%). Compared to control values, the percentage of apoptotic cells increased under both rhARG and Cav treatment $(25 \%)$. These results suggest that $\mathrm{Cav}$ at $0.1 \mathrm{mM}$ range concentration upon rhARG treatment induced only slight progression of apoptosis in PHA-activated PBL. In resting PBL, only spontaneous apoptosis was detected.

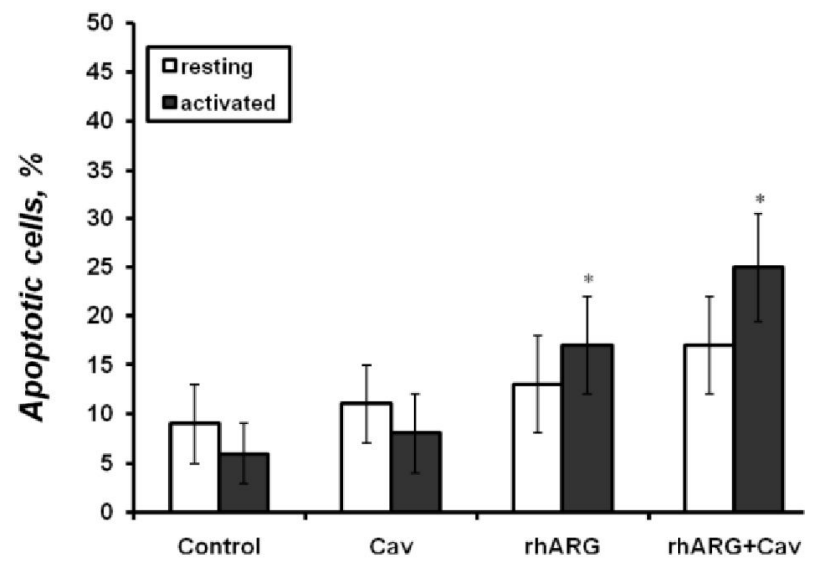

Fig. 3. Effect of the combined rhARG and Cav treatment (72 h) on progression of apoptosis of PHA-activated and resting PBL. Cells were stained with Annexin V and propidium iodide and analyzed in fluorescent microscopy as described in Materials and Methods, ${ }^{*} \mathrm{p} \leq 0.05$ as compared with untreated cells.

The capability for antigen stimulation after different periods of arginine starvation combined with $0.1 \mathrm{mM} \mathrm{Cav}$ treatment was assayed by the reaction of blast-transformation of lymphocytes (RBTL). Cav alone in argininerich medium had no pronounced effect on functional activity of PBL under stimulation by PHA in RBTL (Fig 4). We showed that PBL lost reaction to mitogen stimulation in the absence of arginine in vitro culture medium. Combined action of rhARG with Cav was more immunotoxic than rhARG alone, blocking the proliferation of PBL after stimulation by PHA in RBTL (Fig.4).

We next evaluated the effect of the combined rhARG and $\mathrm{Cav}$ treatment on nitrite production, as nitric oxide production is necessary for normal cooperation of different immune cells [4]. As shown in Fig. 5, PBL cultured in RPMI medium with PHA and rIL-2 in the presence of rhARG were partially affected in nitrite production relative to control cells. Co-culturing with Cav slightly elevated nitrite production in both control and rhARGsupplemented medium. Interestingly, both stimulatory and inhibitory effect of Cav on PHA-activated PBL for healthy individuals was demonstrated $[6,10]$.

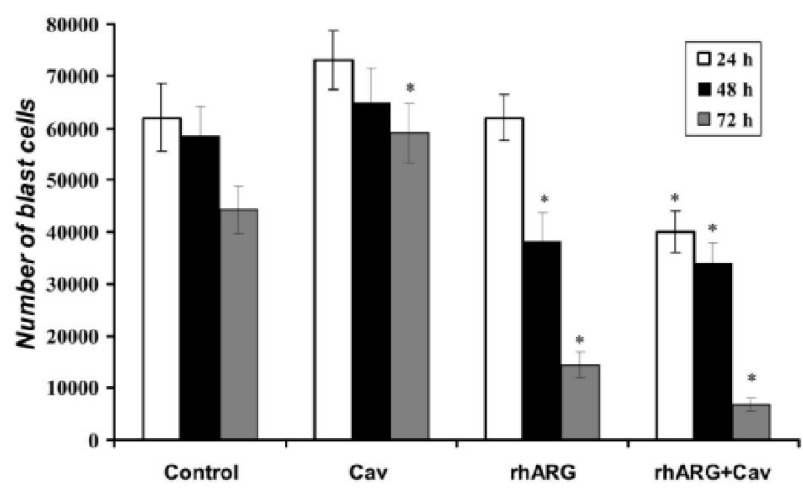

Fig. 4. Effect of combined rhARG and Cav treatment on functional activity of normal human PBL after exposure to rhARG alone or in combination with $0.1 \mathrm{mM}$ Cav in reaction of blast transformation of lymphocytes (RBTL). After the indicated periods of treatment, the medium was changed to complete arginine-rich with PHA and rIL-2 and cells were allowed to grow for additional $96 \mathrm{~h}$. Viable blast cell numbers were determined by the trypan blue exclusion test. ${ }^{\star} \mathrm{p} \leq 0.05$ compared with untreated cells.

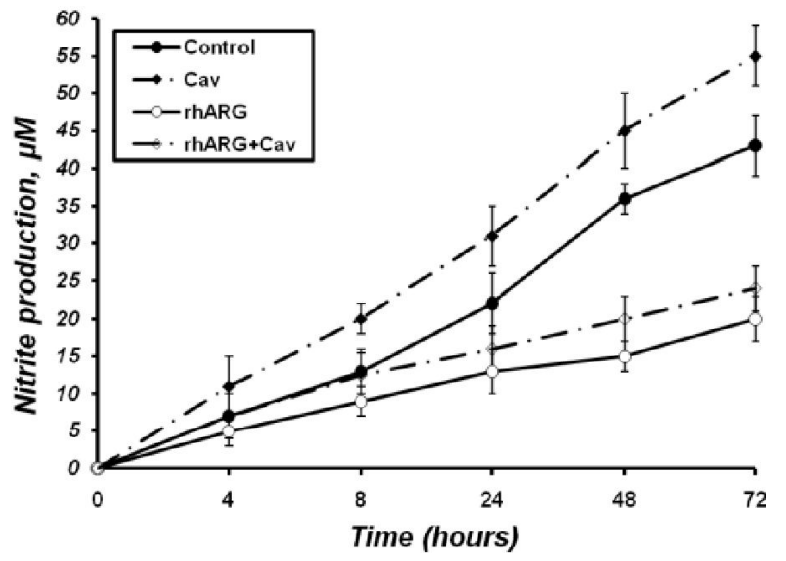

Fig. 5. Time-course nitrite $\left(\mathrm{NO}_{2}\right)$ production in PHA-stimulated PBL. Control medium; Cav - canavanine $0.1 \mathrm{mM}$; rhARG arginase $2 \mathrm{U} / \mathrm{mL}$; rhARG+Cav - arginase $2 \mathrm{U} / \mathrm{mL}$ with $0.1 \mathrm{mM}$ canavanine

\section{CONCLUSIONS}

In this paper we describe for the first time the effect of the combined rhARG and Cav treatment on the viability and functional activity of normal human lymphocytes. We demonstrated that Cav alone has no significant effect on proliferation and functional activity of normal PBL. However, combined rhARG and Cav treatment supressed proliferation of PHA-activated PBL and slightly induced progression of apoptosis in these cells. Normal resting PBL were much more resistant to both drugs' action. We observed that arginine deprivation and $0.1 \mathrm{mM}$ Cav treatment affected the ability to antigen activation and nitrite production in the tested immune cells. These results will be important to consider in the design of further animal 
studies of the combined anticancer treatments based on arginine deprivation.

\section{ACKNOWLEDGEMENTS}

The authors are grateful to N. Igumentseva (Institute of Cell Biology, Lviv, Ukraine) for the invaluable help during experiments described in this study. This study was partially supported by the West Ukrainian BioMedical Research Center (WUBMRC) grant awarded to Chen O.

\section{REFERENCES}

1. Bronte V., Zanovello P.: Regulation of immune responses by L - arginine metabolism. Nat. Rev. Immunol., 5, 641, 2005.

2. Chen O.I. et al.: Effect of nitric oxide donor on viability of human leukemic cells upon arginine deprivation. Studia Biologica, 5, 17, 2011.

3. Dean J.H., Hincks J.R., Remandet B.: Immunotoxicology assessment in the pharmaceutical industry. Toxicol. Lett. 102/103, 247, 1998.

4. Denham S., Rowland I.J.: Inhibition of the reactive proliferation of lymphocytes by activated macrophages. The role of nitric oxide. Clin. Exp. Immunol., 87, 157, 1992.

5. Engeland M., Nieland L.J., Ramaekers F.C.: Annexin Vaffinity assay: a review on an apoptosis detection system based on phosphatidylserine exposure. Cytometry, 31, 1, 1998.

6. Gregory S.H., Sagnimeni A.J., Wing E.J.: Arginine analogues suppress antigen-specific and -non-specific T lymphocyte proliferation. Cell Immunol., 153, 527, 1994.

7. Green M.H., Ward J.F.: Enhancement of human tumor cell killing by $\mathrm{L}$-canavanine in combination with g-radiation. Cancer Res., 43, 4180, 1983.

8. Green L.C., David A.W., Clodowski J.: Analysis of nitrite, nitrite and ISN nitrate in biological fluids. Anal. Biochem., 126, 131, 1992.

9. Jang M.H. et al.: Arginine antimetabolite L -canavanine induces apoptotic cell death in human Jurkat $\mathrm{T}$ cells via caspase- 3 activation regulated by Bcl-2 or Bcl-xLq. Biochem. Biophys. Res. Commun., 295, 283, 2002.

10. Morimoto I. et al.: L-canavanine acts on suppressor-inducer $\mathrm{T}$ cells to regulate antibody synthesis: lymphocytes of systemic lupus erythematosus patients are specifically unresponsive to L-canavanine. Clin. Immunol. Immunopathol., 55, 97, 1990.

11. Rodriguez P.C. et al.: Regulation of $\mathrm{T}$ cell receptor $\mathrm{CD} 3 \zeta$ chain expression by L -arginine. J. Biol. Chem., 277, 21123, 2002.

12. Rodriguez P.C., Quiceno D.G., Ochoa A.C.: L -arginine availability regulates T-lymphocyte cell-cycle progression. Blood, 109, 1568, 2007.

13. Rosenthal G.A.: The biological effects and mode of action of $\mathrm{L}$-canavanine, a structural analogue of $\mathrm{L}$-arginine. Q. Rev. Biol., 52, 155, 1977.

14. Swaffar D.S. et al.: Combination therapy with 5-fluorouracil and L -canavanine: in vitro and in vivo studies. Anticancer Drugs, 6, 586, 1995.

15. Thomas D.A. et al.: Growth inhibition of a rat colon tumor by L -canavanine. Cancer Res., 46, 2898, 1986.

16. Thomas D.A., Rosenthal G.A.: Toxicity and pharmacokinetics of the nonprotein amino acid L -canavanine in the rat. Toxicol. Appl. Pharmacol., 91, 395, 1987.

17. Thomas Yau et al.: A phase 1 dose-escalating study of pegylated recombinant human arginase 1 (Peg-rhArg1) in patients with advanced hepatocellular carcinoma. Invest. New Drugs, 31, 99, 2013.

18. Vynnytska B. et al.: Canavanine augments proapoptotic effects of arginine deprivation in cultured human cancer cells. Anticancer Drugs, 22, 148, 2011.

19. Worthen D.R. et al.: L -Canavanine modulates cellular growth, chemosensitivity and P-glycoprotein substrate accumulation in cultured human tumor cell lines. Cancer Lett., 132, 229, 1998.

20. Yang T-S et al.: A randomised phase II study of pegylated arginine deiminase (ADI-PEG 20) in Asian advanced hepatocellular carcinoma patients. British Journal of Cancer, $103,954,2010$. 